\title{
Web 2.0 als Potential für die Verwaltungs- modernisierung am Beispiel der Fortschreibung einer Verwaltungsvorschrift mittels Wiki
}

\author{
Tanja Röchert-Voigt, Norbert Gronau \\ Lehrstubl für Wirtschaftsinformatik und Electronic Government, \\ Universität Potsdam
}

\section{Einleitung}

Nach Jakobs-Woltering (2008, S. 123) sind $75 \%$ der Kommunalverwaltung Binnenprozesse. Diese internen Prozesse treten meist in den Hintergrund, wenn es darum geht, Verwaltung zu modernisieren. Analysiert und modernisiert werden oftmals nur die verwaltungsexternen Abläufe, die von Bürgern oder Unternehmen angestoßen werden, obwohl gerade die internen Abläufe bereits rein prozentual gesehen viel mehr Potential bieten.

In Zusammenarbeit mit einer Stadtverwaltung wurde eine Wiki-basierte OpenSource-Lösung im Intranet der Verwaltung erfolgreich eingeführt. Mit Hilfe dieses Kollaborationstools des Webs 2.0 wurde das Beteiligungsverfahren zur Fortschreibung einer innerbehördlichen Verwaltungsvorschrift nicht nur medienbruchfrei elektronisch gestaltet, sondern insgesamt transparenter und im Hinblick auf die Auswertung und Erstellung der Beschlussvorlage effektiver und effizienter. Dieses Fallbeispiel soll Potentiale von Web 2.0 im Hinblick auf Rechtsgestaltung und damit einhergehender Entbürokratisierung interner Verwaltungsabläufe aufzeigen.

\section{Ausgangssituation}

In der Stadtverwaltung sollte im Rahmen eines Praxisprojektes der Einsatz von Web 2.0, sowohl vor dem Hintergrund der Zusammenarbeit und Entwicklung einer neuen Allgemeinen Dienstordnung als auch zur Einführung einer neuen Technologie in der Verwaltung getestet werden.

Die Allgemeine Dienstordnung (ADO) der Stadt ist als innerdienstliche Verwaltungsvorschrift oder Dienstanweisung eine Rechtsvorschrift im untergesetzlichen Bereich. Sie stellt ein Regelwerk und Instrument der Verwaltungsführung dar, das für alle Mitarbeiter verbindlich ist. Es werden darin Regeln für Bearbeitungsprozesse und Verantwortlichkeiten festgelegt und damit ein einheitliches, zweck- 
orientiertes, wirtschaftliches Vorgehen der städtischen Verwaltung garantiert. Die ADO gestaltet den Verwaltungsablauf und dient damit der Sicherstellung des Dienst- und Geschäftsbetriebes. In der hierarchischen Organisation der Verwaltung werden die Abläufe aus diesem Grund in immer gleicher Art und Weise abgewickelt. Im Sinne des Rechtsstaatsprinzips trägt das zur Verlässlichkeit, Rechtssicherheit und Transparenz der Organisation Verwaltung und deren Abläufe bei. Verwaltung wird dadurch nachprüfbar und berechenbar (Bogumil und Jann 2009, S. 141).

Das hauptsächliche Problem dieser innerorganisatorischen Regelwerke liegt darin, dass sie - einmal beschlossen - solange gültig sind, bis sie explizit widerrufen oder durch neue Regelungen ersetzt werden. Das hat zur Folge, dass im Lauf der Zeit, gerade aufgrund von Technologieentwicklungen, geänderten Gesetzes- bzw. Rechtslagen oder auch Organisationsveränderungen, eine Vielzahl von nicht mehr zeitgemäßen Regelungen Bestand hat. Das kann schließlich dazu führen, dass Abläufe behindert werden oder sich Dienstanweisungen sogar widersprechen. In diesen Fällen ist eine zeitgemäße und effiziente Abwicklung der Verwaltungsabläufe nicht mehr möglich.

Im konkreten Fallbeispiel sollte aufgrund festgestellten Änderungsbedarfs die ADO grundlegend überarbeitet bzw. fortgeschrieben werden. Als Basis sollte dafür ein Regelungswerk mit Rahmencharakter geschaffen werden, das durch weitere Dienstanweisungen für bestimmte, sich regelmäßig ändernde Bereiche wie z. B. IT, Datenschutz, Personal, Organisation in zukünftigen Schritten konkretisiert werden konnte.

Im Rahmen der Anforderungsanalyse wurde zunächst das herkömmliche Verfahren zur Erstellung bzw. Fortschreibung einer innerdienstlichen Verwaltungsvorschrift auf Basis von Dokumenten, ergänzt durch Experteninterviews aufgenommen. Ein Entwurf einer Rahmenregelung, in Abgrenzung zur bestehenden Regelung als Allgemeine Dienst- und Geschäftsordnung (ADGO) bezeichnet, war bereits von der dafür zuständigen Stelle der Stadtverwaltung gefertigt worden. Herkömmlicherweise wird dieser Entwurf dann im innerbehördlichen Beteiligungsverfahren bearbeitet. Innerbehördliche Beteiligungsverfahren sind bei allen Vorlagen notwendig, die zur Beschlussfassung dem Beschlussgremium vorgelegt werden. Dieses Verfahren ist nicht gesetzlich statuiert, sondern als Bestandteil des innerdienstlichen Arbeitsablaufs lediglich innerorganisatorisch festgelegt. Herkömmlicherweise wird der Entwurf der ADGO daher auf dem festgelegten verwaltungsorganisatorischen Weg den einzelnen Abteilungen zugeleitet. Die Fachbereichs- und Bereichsleiter der Abteilungen erhalten so die Möglichkeit, Stellungnahmen und Änderungswünsche zur ADGO abzugeben. Darüber hinaus wird der Personalrat beteiligt. Die Stellungnahmen und Änderungswünsche der Führungskräfte gelangen dann in unterschiedlichen Formen - als handschriftlich überarbeitetes Papierdokument, als digital überarbeitetes Gesamtdokument oder als mit der „Überarbeiten-Funktion“ bearbeitetes digitales Dokument - mit durchschnittlich 25 Änderungen pro Dokument an die zuständige Stelle zurück. Die zuständige 
Stelle hat so einerseits eine Fülle von Papierdokumenten und andererseits eine Fülle von E-Mails auszuwerten und zu einem erneuten ADGO-Entwurf zusammenzustellen, der gegebenenfalls erneut zur Abstimmung mit betroffenen oder beteiligten Abteilungen in die Beteiligungsrunde geleitet wird. Liegt schließlich ein finaler, abgestimmter ADGO-Entwurf vor, wird dieser gedruckt und gelangt auf dem Dienstweg zur Beschlussvorlage zum Oberbürgermeister. Nach Unterschrift und Ausfertigung wird die neue Dienstanweisung, insbesondere im Intranet oder in Rundschreiben, bekanntgemacht und tritt in Kraft.

Eine direkte Stellungnahme zu Änderungswünschen anderer Abteilungen oder eine Zusammenarbeit der Abteilungen im Hinblick auf eine abgestimmte Erstellung der ADGO ist in dem herkömmlichen Beteiligungsverfahren aufgrund der hierarchischen Struktur nicht vorgesehen. Das Verfahren ist nach Aussagen der Beteiligten insgesamt nicht nur zeitaufwendig, sondern auch für alle sehr arbeitsintensiv.

\section{Einsatzpotential von Web 2.0 für interne Prozesse der Verwaltung}

Es stellte sich die Frage, ob Web 2.0-Technologien und -Anwendungen mit Ihren Grundsätzen und Prinzipien geeignet sind, dieses Beteiligungsverfahren zur Rechtsetzung in der hierarchisch organisierten Verwaltung im Hinblick auf Zeit und Arbeitskraft effizienter zu gestalten und so einen Beitrag zur innerbehördlichen Entbürokratisierung und Transparenz leisten können. Auf Einsatzpotentiale moderner IuK-Technologien im Gesetzgebungsverfahren hat zuletzt von Lucke (2009, S. 10) hingewiesen.

Der Begriff des Web 2.0 wurde 2005 maßgeblich durch Tim O’Reilly (2005, S. 1-16) geprägt. Er steht einerseits für ein offenes Technologiekonzept, im Rahmen dessen Personen, Daten und Dinge verknüpft werden können und andererseits für ein Organisationskonzept, das hauptsächlich auf Vernetzung von Personen, Organisationen und Wissen basiert (BITKOM 2008, S. 4; Kaczorowski 2008, S. 132) und zum Ziel hat, das Arbeiten effizienter und effektiver zu gestalten. Anstelle des Konsumenten von Wissen wird bei Web 2.0 vom sogenannten Prosumenten gesprochen (Habbel und Huber 2008, S. 1; Lattemann et al. 2009, S. 20 ), der sich nicht nur dessen bedient, was er an Inhalten im Internet vorfindet (Konsument), sondern darüber hinaus selbst Inhalte aktiv gestaltet und damit auch zum Autor der Inhalte wird (Riemer 2009, S. 13). Web 2.0 bedeutet ferner, dass Inhalte organisationsübergreifend und hierarchiefrei erschlossen und fortentwickelt werden können (Kaczorowski 2008, S. 133). Diese Art der Fortentwicklungsmöglichkeit von Inhalt schließt die Möglichkeit zum Review von bereitgestelltem Inhalt durch andere Nutzer mit ein und macht so den Kern des durch Web 2.0 entstehenden sozialen Netzwerkes aus. 
Kennzeichnend für Web 2.0-Anwendungen sind also zusammenfassend browserbasierte Anwendungen, mit denen verschiedene Datenquellen beliebig durch Nutzerpartizipation kombiniert, ergänzt und neu zusammengeführt werden können. Beispiele für die Vielfältigkeit von Webanwendungen sind Google, Flickr und Twitter, um nur einige zu nennen. Social Tagging oder Bookmarks (z. B. Delicius, Digg oder ShortNews) sind beispielsweise Anwendungen zum Zusammenführen und Gestalten von Datenquellen. Und als berühmtestes Beispiel für Nutzerpartizipation und Zusammenarbeit in Form eines Wikis im Internet sei Wikipedia genannt. Im Unterschied zum Einsatz dieser Web2.0-Technologien und -Anwendungen im privaten und privatwirtschaftlichen Bereich sind hier verwaltungsspezifische Anforderungen zu berücksichtigen, damit letztlich für den Verwaltungsbetrieb effektivere und effizientere Prozesse entstehen. Die im Verhältnis zu privaten oder zu privatwirtschaftlichen Organisationen grundlegende verwaltungsspezifische Anforderung ist der streng hierarchische Aufbau der Verwaltung, der in der Regel durch Linien- oder Stab-Linien-Organisation gekennzeichnet ist. Es gibt hier einen eindeutigen Dienstweg und eine klare Kompetenzverteilung, sowie u.a. eine klare Struktur für Kommunikation (Bogumil und Jann 2009, S. 140). Die klare Struktur für Kommunikation auf dem Dienstweg birgt aber auch den entscheidenden Nachteil erschwerter Kommunikation gleichrangiger Stellen und verhindert zeitnahe Abstimmungsprozesse.

Für die Unterstützung der Zusammenarbeit im innerbehördlichen Beteiligungsverfahren der LHP wurde ein Wiki ausgewählt. CSCW-Systeme unterstützen die Zusammenarbeit ebenso wie Wikis. Im Gegensatz zu diesen benötigen sie aber für eine Adoption vor allem Zeit und verursachen in der Regel Kosten (Klein und Krcmar 2001, S. 10). Dieses Projekt stand unter der Prämisse des Testens von Web 2.0-Technologien in der Verwaltung und der Nutzung von OpenSourceSoftware. Vor diesem Hintergrund wurden CSCW-Systeme nicht in die Auswahl einbezogen.

Ein Wiki kann sowohl im Internet als auch in lokalen Netzwerken eingesetzt werden. Es ist ein Kollaborations- und Wissensmanagementwerkzeug (Müller und Dibbern 2006, S. 45), mit dem Inhalte oder Erfahrungen gesammelt oder Informationen ausgetauscht werden können. Von Lucke (2008, S. 139) beschreibt Wikis als offene Content-Management-Systeme (CMS), deren Inhalte schnell und einfach gelesen und bearbeitet werden können. Im privaten Bereich werden Wikis in der Regel zur kollaborativen Sammlung von Wissen und Erfahrung genutzt (vgl. Wikipedia). Zweck von Wikis in Unternehmen ist die kollaborative Erstellung von Dokumenten, die Erfassung von Softwarefehlern oder die Ideensammlung (Müller und Gronau 2008, S. 15). Wikis sind einfach zu bedienen und verursachen, insbesondere als OpenSource-Software, keine zusätzlichen Anschaffungskosten.

Das innerbehördliche Beteiligungsverfahren der Stadt als solches verfolgt gerade den Zweck, die Beweggründe und Argumente der beteiligten Fachverfahren für Änderungen von Vorschriften in Erfahrung zu bringen. Es ist ein verwaltungsinternes Instrument für die Umsetzung von rechtlichem Gehör als Ausprägung der 
Rechtsstaatlichkeit. Ein aktiver Informationsaustausch ist hier Ziel und Zweck des Verfahrens. Daher eignet sich das Kollaborationstool Wiki grundsätzlich, um ein solches Verfahren zu unterstützen. Da das Wiki ein sozio-technisches System ist (Koch et al. 2009, S. 47), genügen der Organisation angepasste technische Voraussetzungen nicht allein, vielmehr lebt das Wiki vom „Mitmachen“ und der Eigeninitiative der Nutzer (BITKOM 2008, S. 22; Lucke (2008), S. 140). Der Erfolg eines Wikis in der Verwaltung ist daher maßgeblich von der Initiative der Verwaltungsmitarbeiter abhängig.

Die Verwaltung ist als Teil der Exekutive an Vorschriften gebunden. Die Abläufe in der Verwaltung sind wie oben beschrieben von hierarchischen Strukturen, Dienstanweisungen und traditionellen Denkweisen geprägt. Während im privatwirtschaftlichen Sektor Web2.0-Technologien und -Anwendungen schon stark genutzt werden, findet diese Nutzung im öffentlichen Verwaltungsalltag noch kein Pendant. Insofern lag die Herausforderung auch darin, dieses Kollaborationsinstrument Wiki in diesen Kontext nutzenbringend zu integrieren.

In einem Workshop wurden zunächst die technischen Anforderungen der Stadt und die Details für die Rechtevergabe festgelegt. Dabei konkretisierten sich folgende Schwerpunkte, die der Einsatz eines Wikis in der Stadtverwaltung erfüllen musste:

- Benutzeroberfläche in deutscher Sprache

- Abbildung der ADGO durch die Übernahme der bereits durch die Dienst- und Geschäftsordnung gegebenen Struktur

- Intuitive Struktur, Ein- und Ausgabe mittels WYSIWYG-Editor

- Möglichkeit der gleichzeitigen Bearbeitung durch Nutzer

- Änderungshistorie zum Nachvollziehen der Aktivitäten im Wiki

- Hinzufügen und Nutzen von Anlagen, Formblättern und ähnlichen Dokumenten, ggf. über Up- und Download Funktionalität der Oberfläche

- Navigation zwischen einzelnen Abschnitten über Links

- Verweise auf verwandte Ordnungen, Gesetzestexte, Glossare über Links

- Rechtekonzept (Wer darf lesen, schreiben, ändern?)

Auf Basis der Anforderungsanalyse wurde ein Kriterienkatalog erstellt, der den Ausgangspunkt für ein intensives Auswahl- und Testverfahren, sowie Testinstallationen verschiedenster Wiki-Lösungen bildete. Als die für das innerbehördliche Beteiligungsverfahren geeignete Lösung ergab sich schließlich ein Open-Source MediaWiki mit einer Open-Source MySQL-Datenbank. Bei der Entscheidungsfindung wurden Marktrecherchen und Erfahrungen aus Pilotprojekten, sowie die positive Testinstallation und -präsentation einbezogen. Ein wichtiger Grund im Hinblick auf die Entscheidung für eine MediaWiki-Lösung war darüber hinaus die Tatsache, dass diese Software ihre Skalierbarkeit bereits als Basis von Wikipedia 
bewiesen hat. Sie wird durch die gemeinnützige MediaWiki Stiftung betreut und unterstützt und ist aufgrund dessen gut dokumentiert. Von einer aktiven Weiterentwicklung dieser Software konnte daher ausgegangen werden. Außerdem war eine Anpassung der Software auf die Bedürfnisse der LHP nur in geringem Maß erforderlich.

Neben den Funktionen „Lesen“ und „Bearbeiten“ ist bei der MediaWikiSoftware auch eine „Diskussion“ jeweils zu den einzelnen Vorschriften möglich. Die beteiligten Geschäftsbereichsleiter und Fachbereichsleiter haben so die Möglichkeit, nicht nur den Entwurf zu lesen, sondern gleich die ihrer Ansicht nach notwendigen Änderungen einzufügen. Für eine benutzerfreundliche Handhabung der „Bearbeiten“-Funktion durch die beteiligten Leiter wurde ein WYSIWYGEditor (wikEd) angepasst. Dieser Editor hat den entscheidenden Vorteil, dass Programmier- oder Syntaxkenntnisse nicht erforderlich sind und die Verwaltungsmitarbeiter Inhalte des ADGO-Entwurfs so ändern und ergänzen können, wie sie es mit den jeweiligen Textverarbeitungsprogrammen aus dem Verwaltungsalltag gewohnt sind. Durch die Versionsverfolgung und -kontrolle ist jederzeit durch jeden ein Vergleich zu vorherigen Versionen des Entwurfs möglich. Sollten die gewünschten Änderungen eines Bereichsleiters keinen Anklang finden, kann die dazu befugte Person im Rahmen der Versionskontrolle die Änderungen im Entwurf auch jederzeit in einen vorherigen Arbeitsstand zurückstellen.

\section{Arbeit mit dem Wiki und Evaluationsergebnisse}

Die Anmeldung im Wiki erfolgt aufgrund der verwaltungsinternen LDAPAnbindung über die bekannten Benutzernamen und Passwörter. Grundsätzlich kann jeder Verwaltungsmitarbeiter sich über den Stand des Entwurfs der ADGO informieren. Über die Individuelle Anmeldung erhält aber jeder Nutzer des Wikis nur die ihm aufgrund des Rechtekonzepts zugewiesenen Befugnisse, z. B. nur zum Lesen oder darüber hinaus auch zum Bearbeiten bzw. zur Versionskontrolle. Nach der erfolgreichen Anmeldung erscheint die Hauptseite. Die jeweiligen Rubriken „Inhalt der ADGO“, „Glossar zur ADGO“, „Weitere Dokumente“ und „Schulungsunterlagen" sind auch auf der Hauptseite zu finden und jeweils als Link zu den entsprechenden inhaltlichen Seiten ausgestaltet. Inhalte der ADGO sind auch über die Suchenfunktion auf der linken Navigationsleiste auffindbar. Die Rubrik „Glossar" enthält darüber hinaus alphabetisch aufgelistet verlinkte Erläuterungen von Begriffen der ADGO. Das Glossar ist änder- und erweiterbar. Die Rubrik „Weitere Dokumente“ enthält Mustervorlagen, auf die im Rahmen der ADGO hingewiesen wird. Hier können ebenfalls weitere Vorlagen oder Anlagen ergänzt werden. Die Schulungsunterlagen zum Umgang mit dem Wiki liegen unter der gleichnamigen Rubrik als anschauliche Anleitung zum Nachschlagen, die ebenfalls jederzeit änderbar ist. 


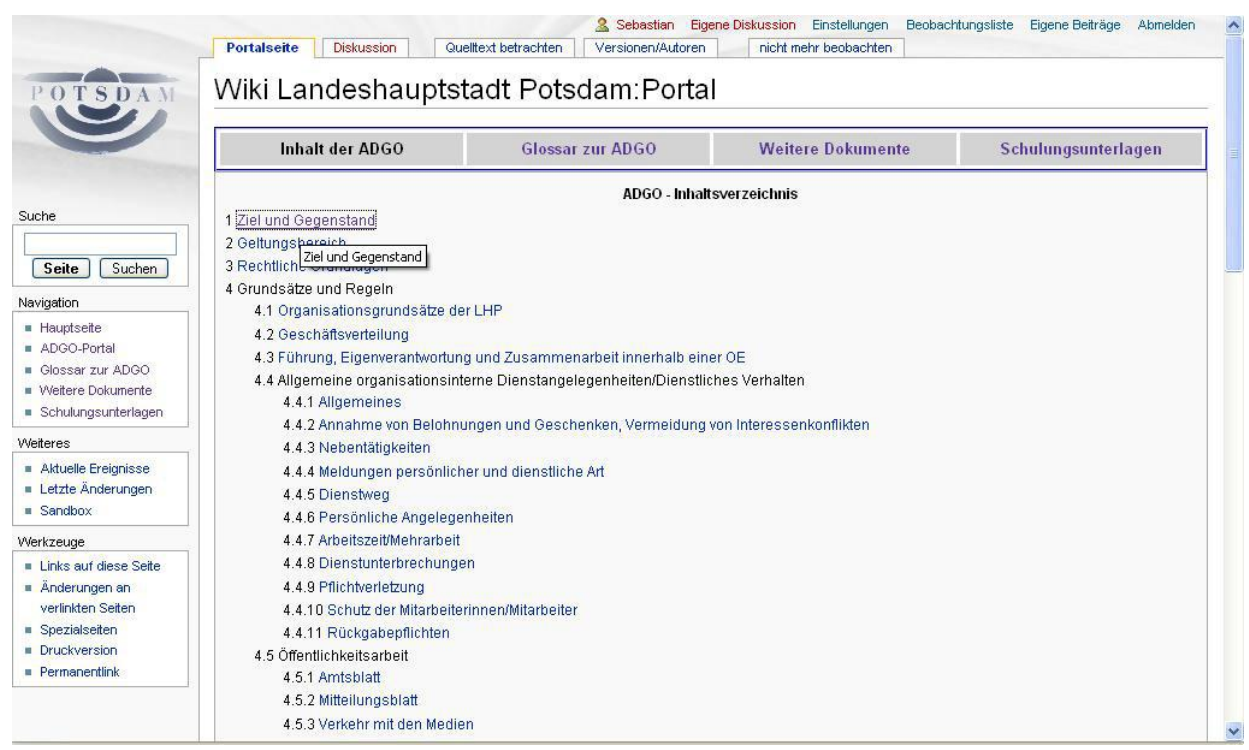

Abbildung 1: Screenshot Wiki-Startseite mit Gliederung ADGO

Die bearbeitbaren Inhalte der ADGO erreicht der berechtigte Nutzer jeweils über die als Link ausgestalteten bezifferten Überschriften der Gliederung. Nach anklicken der gewünschten Überschrift kann über den MediaWiki-typischen „Bearbeiten“-Reiter der Inhalt geändert oder ergänzt werden.

Zur besseren Orientierung des Verwaltungsmitarbeiters an der inhaltlichen Gliederung der ADGO findet sich in der erste Zeile nach der Überschrift („Ziel und Gegenstand“) der Navigationsweg („Inhaltsverzeichnis, Ziel und Gegenstand"). So ist jederzeit ersichtlich, welcher Inhalt von welcher Vorschrift unter welcher Oberüberschrift gerade bearbeitet wird.

Darüber hinaus kann im Rahmen der „Diskussion“ zu jedem inhaltlichen Gliederungspunkt auf spezifische Argumente für die jeweils gewollte Änderung hingewiesen und zu anderen Argumenten Stellung bezogen werden.

Für bestimmte Fachbereiche sind bestimmte Abschnitte der ADGO relevanter als andere. Diesbezügliche Veränderungen können über die Funktion des Beobachtens aktuell verfolgt werden, so dass auch die Möglichkeit zur sofortigen Stellungnahme gegeben ist, wodurch der Weg über die zuständige Stelle, deren Erstellung eines geänderten Zwischenentwurfs und einer erneuten Stellungnahme eine erhebliche Abkürzung erfährt und eine Unmittelbarkeit erzeugt wird, die in dem bisherigen Verfahren aufgrund des Dienstweges nicht vorgesehen war.

Da die Änderungswünsche und -gründe nunmehr auch in einheitlicher digitaler Form vorliegen, kann medienbruchfrei insgesamt ein angepasster ADGO-Entwurf durch die zuständige Stelle gewonnen werden, was gegenüber der herkömmlichen Verfahrensweise auch eine deutliche Verbesserung darstellt. Über die Versionskon- 
trolle kann eine Qualitätssicherung, gegebenenfalls auch durch Zurücksetzen in einen vorherigen Bearbeitungsstand, bereits während der Zeit der Stellungnahmen erfolgen. Im Gegensatz zu der herkömmlichen Verfahrensweise besteht nunmehr auch die Möglichkeit, dass alle nutzungsberechtigten Mitarbeiter innerhalb eines bestimmten, vorgegebenen Zeitrahmens ihre Änderungswünsche einpflegen und auch zu Änderungswünschen der anderen Mitarbeiter Stellung beziehen können. Hier wurde so ein interaktiver Kollaborationsbereich anstelle des herkömmlichen nach- und nebeneinander Arbeitens geschaffen. Damit aus technischer Sicht keine Speicherkonflikte bei der gleichzeitigen Bearbeitung desselben Inhalts entstehen, ist ein Inhalt, der gerade von einem Mitarbeiter bearbeitet wird, solange gesperrt, bis seine Änderungen gespeichert sind. Erst dann kann eine weitere Bearbeitung durch einen anderen Mitarbeiter erfolgen.

Insgesamt wird die ADGO-Fortschreibung nach Aussagen der Beteiligten durch das Kollaborationswerkzeug Wiki auch in zeitlicher Hinsicht deutlich effizienter.

Nach einer kurzen Testphase mit zehn zuvor geschulten Mitarbeitern in einer ersten Stufe der Wiki-Nutzung, konnten insbesondere folgende Erfahrungen mittels Fragebogen und Interview gewonnen werden:

Alle Mitarbeiter standen dem Einsatz eines solchen neuen Arbeitswerkzeugs grundsätzlich aufgeschlossen gegenüber. Diese grundlegende Voraussetzung erleichterte die Wiki-Einführung. Ferner gestaltete sich die Arbeitsweise mit dem Wiki aufgrund des Editors nach Einschätzung der Beteiligten benutzerfreundlich, weil sie dem Umgang mit den gewohnten Textverarbeitungsprogrammen entsprach. Konkret würden sieben der zehn Mitarbeiter der ersten Stufe mit dem Tool arbeiten wollen. Die ablehnende Haltung von drei Mitarbeitern resultierte aus einer generellen Unsicherheit im Umgang mit der rein digitalen Verwaltungsarbeit am Bildschirm. Inwieweit die hierarchische Struktur und die Fehlerkultur Einfluss auf Unsicherheiten haben, wird weiter untersucht. Gegebenenfalls könnten auch intensivere Schulungsmaßnahmen einen geübteren Umgang mit den neuen Techniken bewirken.

In einer zweiten Stufe wirkten 30 Führungskräfte aus dem Bereich Personal und Organisation, sowie Mitarbeiter aus dem Personalrat mit. Diese hatten sich auf Anfrage sämtlich freiwillig bereit erklärt und standen der Nutzung aufgeschlossen gegenüber. Auf Basis der erstellten Schulungsmaterialien wurden die Mitarbeiter der zweiten Stufe von den zuvor geschulten Mitarbeitern in den Umgang mit dem Wiki eingewiesen und haben sich dann ebenso intensiv bei der Fortschreibung der ADGO eingebracht. Hemmnisse, Hindernisse oder Probleme in Bezug auf die Mitwirkungsbereitschaft gab es auch in dieser zweiten Stufe der WikiNutzung nicht. Es konkretisierte sich allerdings, dass die gewohnte herkömmliche Arbeitsweise mit der Papierakte die neue Arbeitsweise mit dem Wiki noch überschattet, weil sie sich rein digital gestaltete und daher im Verwaltungsalltag ungewohnt war. Aufgrund der Rechtevergabe im Wiki war es nun auch nicht mehr möglich, dass der mit der Bearbeitung betraute Mitarbeiter die Aufgabe auf einen 
anderen übertrug. Die Arbeit mit dem Wiki bewirkte in diesem Fall daher ein sich Bewusstmachen über Aufgaben und Aufgabenzuständigkeiten. Die bewusste Rechtevergabe hatte damit Einfluss auf die konkrete Umsetzung festgeschriebener Aufgabenzuständigkeiten und mithin auf die gelebte Verwaltungskultur.

Positiv wurde von den Beteiligten hervor gehoben, dass es aufgrund des Wikis möglich sei, schnell auf die Bearbeitungsergebnisse anderer zuzugreifen, diese zu vergleichen und dazu Stellung zu beziehen. Eigene Intentionen konnten so unmittelbar mitgeteilt werden, ohne dass der vorgeschriebene Dienstweg des herkömmlichen Beteiligungsverfahrens über die zuständige Stelle und die Stellungnahme zu einem geänderten Entwurf eingehalten werden musste.

\section{$5 \quad$ Fazit und Ausblick}

Insgesamt wird das Wiki von den befragten Beteiligten in der Stadtverwaltung als sinnvolles und übersichtliches Tool für eine praktikable und schnelle Bearbeitung der ADGO erachtet. Großes Einsparungspotential ergibt sich insbesondere aus dem Wegfall der „E-Mail-Flut“ mit den unterschiedlichsten Stellungnahmen, sowie aus dem Wegfall der „Papierberge“. Durch das Vorliegen eines einheitlichen digitalen Dokuments wird sowohl die Auswertung als auch die abschließende Erstellung einer Beschlussvorlage durch die zuständige Stelle wesentlich erleichtert. Da das Wiki als sozio-technisches System insbesondere von der Eigeninitiative der Mitarbeiter lebt, ist es notwendig, dass eine entsprechende Organisationsstruktur und Kultur in der Verwaltung gegeben ist, die diese Eigeninitiative fördert. In der Stadtverwaltung konnten die Mitarbeiter sämtlich auf freiwilliger Basis zur Mitarbeit gewonnen werden. Anreizsyteme mussten aufgrund dieser Verwaltungskultur nicht geschaffen werden. Der Einfluss der hierarchischen Struktur auf die Eigeninitiative der Mitarbeiter stand im Fallbeispiel deswegen nicht zur Diskussion, wird im Rahmen weiterer Forschungsaktivitäten aber ebenso wie der Einfluss der hierarchischen Struktur und der Fehlerkultur auf Unsicherheiten im Umgang mit dem neuen Medium eingehender fokussiert.

Darüber hinaus konnte festgestellt werden, dass die bewusste Rechtevergabe im Wiki derart Einfluss auf die gelebte Verwaltungskultur hat, dass die vorgegebene Aufgabenzuständigkeit nicht delegiert werden kann. Die Emergenz möglicher Schattenprozesse in hierarchischen Strukturen und deren Veränderung durch technisch gestützte Zugriffsmedien gilt es im Rahmen der weiteren Forschung zu untersuchen.

Aufgrund dieses insgesamt positiven Ergebnisses und erfolgreichen Tests wird in der Stadtverwaltung angestrebt, zukünftig alle Beteiligungsverfahren generell elektronisch mittels eines Wikis durchzuführen. Vor dem Hintergrund der notwendigen Beachtung von Standards, insbesondere im Hinblick auf die Pflege und Betreuung der Datenbank und der gewünschten Vermeidung von Schnittstellen, wird zukünftig aber ein integriertes Anwendungskonzept angestrebt, das neben 
dem Wiki weitere Möglichkeiten (z. B. Blogs, Foren, Instant Messaging, E-Mail usw.) bietet.

Das Fallbeispiel belegt, dass Web 2.0-Anwendungen wie das Wiki im Ergebnis geeignet sind, ein Beteiligungsverfahren im Rahmen innerdienstlicher Rechtsetzung in der hierarchisch organisierten Verwaltung effektiv und effizient zu gestalten. Der Einsatz eines Wikis zur Fortschreibung einer innerdienstlichen Verwaltungsvorschrift ist daher ein neuer, innovativer Ansatz in Richtung digitale Verwaltung, Abbau innerbehördlicher Bürokratie und Schaffung von innerbehördlicher Transparenz.

\section{Literatur}

BITKOM (2008) Web 2.0 für die öffentliche Verwaltung. Grundzüge, Chancen, Beispiele und Handlungsvorschläge. Http:// www.bitkom.org/files/dokuments/Web 2.0 fuer die oeffentliche verwaltung.pdf. Abruf am 2009-09-14.

Bogumil J, Jann W (2009): Verwaltung und Verwaltungswissenschaft in Deutschland. Verlag für Sozialwissenschaften, Wiesbaden.

Habbel FR, Huber A (2008) Bürger gestalten Kommunalpolitik. In: Habbel FR, Huber A (Hrsg.) Web 2.0 für Kommunen und Kommunalpolitik - Neue Formen der Öffentlichkeit und der Zusammenarbeit von Politik, Wirtschaft, Verwaltung und Bürger. VWH, Boizenburg.

Jakobs-Woltering P (2008) Web 2.0-Anwendungen und ihre Einsatzmöglichkeiten. In: Habbel FR, Huber A (Hrsg.) Web 2.0 für Kommunen und Kommunalpolitik - Neue Formen der Öffentlichkeit und der Zusammenarbeit von Politik, Wirtschaft, Verwaltung und Bürger. VWH, Boizenburg.

Kaczorowski W (2008) Soziale Netzwerke in Behörden. In: Habbel FR, Huber A (Hrsg.) Web 2.0 für Kommunen und Kommunalpolitik - Neue Formen der Öffentlichkeit und der Zusammenarbeit von Politik, Wirtschaft, Verwaltung und Bürger. VWH, Boizenburg.

Klein A, Krcmar H (2001) E-Learning und Collaborative Commerce - was sie von der CSCW - Forschung lernen können. Wirtschaftinformatik Proceedings 2001. Paper 24. http://aisel.aisnet.org/wi2001/24. Abruf am 25.11.2009

Koch M, Ott F, Richter A (2009) Wikis und Weblogs im Wissens- und Innovationsmanagement. In: Riemer K; Strahringer S (Hrsg.) eCollaboration. HMD- Praxis der Wirtschaftsinformatik 267 (6):47-55

Lattemann C, Stieglitz S, Kupke S (2009) Deutsche Unternehmen auf dem Weg zum Web 2.0? In Riemer K; Strahringer S (Hrsg.) eCollaboration. HMDPraxis der Wirtschaftsinformatik 267 (6): 18-26 
Leuf B, Cunningham W (2001) The Wiki-Way - Quick Collaboration on the Web. Addison-Wesley, New York.

Lucke J von (2008) Wikis in der Verwaltung. In: Hass, B.H. (Hrsg.) Web 2.0: Neue Perspektiven für Marketing und Medien. Springer, Berlin

Lucke J von (2009) Beiträge der Wirtschaftsinformatik zum eGovernment. In: Hofmann J, Reich S (Hrsg.) eGovernment. HMD- Praxis der Wirtschaftsinformatik 265 (2):7-18

Müller C, Dibbern P (2006) Selbstorganisiertes Wissensmanagement in Unternehmen auf Basis der Wiki-Technologie - ein Anwendungsfall. In: Hildebrandt K, Hoffmann J (Hrsg.) Social Software. HMD - Praxis der Wirtschaftsinformatik, $252(6): 45-54$.

Müller C, Gronau N. (2008) Wikis. In: Back A, Gronau N, Tochtermann K (Hrsg.) Web 2.0 in der Unternehmenspraxis. Grundlagen, Fallstudien und Trends zum Einsatz von Social Software. Oldenbourg, München.

O'Reilly T (2005) What is Web 2.0. Design Patterns and Business Models for the next Generation Software. Http://oreilly.com/web2/archive/what-is-web20.html. Abruf am 2009-09-16

Riemer K (2009) eCollaboration: Systeme, Anwendungen und aktuelle Entwicklungen. In: Riemer K; Strahringer S (Hrsg.) eCollaboration. HMD- Praxis der Wirtschaftsinformatik 267 (6):7-17 